\title{
The price of portfolio selection under tail conditional expectation with consumption cost and transaction cost
}

\author{
Bright Okore Osu $^{\dagger, *}$, Silas Abahia Ihedioha ${ }^{\dagger}$ and Enyinnaya Ekuma-Okereke ${ }^{\ddagger}$ \\ ${ }^{\dagger}$ Department of Mathematics, Abia State University, P.M.B. 2000, Uturu, Nigeria \\ ${ }^{\ddagger}$ Department of Mathematics, Rhema University, Aba, Nigeria
}

Received: May 04, 2013; Accepted: July 09, 2013

Copyright (c) 2013, Afrika Statistika. All rights reserved

\begin{abstract}
One of the ways of managing the risk that can arise from the changes in the relationship between assets and liabilities is by asset-liability management. Recently, Value-at-risk (VaR) and tail conditional expectation (TCE) have also emerged as standard tools for measuring and controlling the risk of trading portfolios. The limits of TCE can be transformed into the limits of VaR and conversely in some dynamical setting, TCE is preferable to VaR for being coherent. In this paper we obtain a portfolio selection model for an institution's assets- liabilities under the TCE with consumption cost and transaction cost. A set of partial differential equations are derived and closed form solution proffered, when there is no transaction cost.

Résumé. Jusqu'à récemment, les mesures de risque dénommées Valeur Au Risque (VaR) et l'Espérance Conditionnelle de Queue (ECQ), étaient utilisées pour évaluer et controler les risques en gestion de portefeuille. Ces deux mesures sont équivalentes dans les cas limites. De plus, dans une approche dynamique, l'ECQ est préférable à la VaR en raison notamment de sa cohérence. Une autre approche de la gestion des portefeuille concerne l'évaluation du risque potentiellement dû aux changements qui peuvent intervenir dans la relation entre les actifs et les passifs, communément dénommée gestion de l'actif-passif. Dans ce papier, nous proposons un modèle de sélection de portefeuille d'actifs-passifs d'une institution sous contrôle du TCE en présence des coûts de consommation et de transaction. Une famille d'équations différentielles partielles est proposée et une solution fermée est trouvée, lorsqu'il n'y a pas de coût de transaction.
\end{abstract}

Key words: Risk management; Tail conditional expectation; Asset-liability control; Value-at-risk; consumption cost; Transaction costs.

AMS 2010 Mathematics Subject Classification : Primary: 91G10, 37NA; Secondary: D92, C61.

${ }^{*}$ Corresponding author Bright Okore Osu: megaobrait@yahoo.com

Silas Abahia Ihedioha : silasihedioha@yahoo.com

Enyinnaya Ekuma-Okereke : enyishine@yahoo.com 
B.O. Osu, S.A. Ihedioha and E. Ekuma-Okereke, Afrika Statistika, Vol. 8, 2013, pages 545-559.

The price of portfolio selection under tail conditional expectation with consumption cost and transaction cost.

\section{Introduction}

Financial risk management is vital to the survival of financial institutions and the stability of the financial system. A fundamental task in risk management is to measure the risk entailed by a decision, such as the choice of a portfolio (Lan et al., 2010). Recently, the substitution of variance as a risk measure in the standard Markowitz (1952) mean-variance problem has been emphasized, because it makes no distinction between positive and negative deviations from the mean. Variance is a good measure of risk only for distributions that are (approximately) symmetric around the mean such as the normal distribution or more generally, elliptical distributions (Frey and Embrechts, 2006). In cases such as in portfolio containing option as well as credit portfolio (i.e wealth distributions that are highly skewed), it is reasonable to consider asymmetric risk measures since individuals are typically loss averse. Ait-Sahalia (2001) studied asset allocation when the conditional moments of returns are partly predictable. Rather than first model the return distribution and subsequently characterize the portfolio choice, the determined directly the dependence of the optimal portfolio weights on the predictive variables. Asset-liability control is a means of managing the risk that can arise from changes in the relationship between asset and liabilities.

In this regard, Value-at-Risk, VaR, a downside (negative) risk measure, has also emerged as the industry standard with regulatory authorities enforcing its use in risk measurement and management, Jorion (2001). Despite its widespread acceptance, VaR is known to possess unappealing features. Artzner et al. (1999) proposed an axiomatic foundation for risk measures, by identifying four properties that a reasonable risk measure should satisfy and provided a characterization of the risk measures satisfying these properties, which they called coherent risk measures. Tail conditional expectation TCE is one of such so-called coherent risk measures (Rockafellar and Uryasev, 2001). Going by these axioms, VaR is not coherent. TCE provides a more conservative measure of risk than VaR for the same level of degree of confidence (Landsman and Valdez, 2003). Therefore, the TCE is preferable to the VaR in many applications and has recently received growing attentions in the insurance and finance literature.

Let risk $Z$ be a non-negative random variable with cumulative distribution $F$, where $Z$ may be referred to as a claim for an institution's asset or liability. Given $0<\alpha<1$, then $z_{\alpha}$, determined by $\bar{F}\left(z_{\alpha}\right)=1-F\left(z_{\alpha}\right)=\alpha$ and denoted by $\operatorname{VaR}_{Z}(1-\alpha)$ is called the value at risk $\mathrm{VaR}$ with a degree of confidence $1-q$. The conditional expectation of $Z$ given by $Z>z_{\alpha}$ denoted by $T C E_{Z}=\mathbb{E}\left(Z \mid Z>z_{\alpha}\right)$ is called the a tail conditional expectation (TCE) of $Z$ at $\operatorname{VaR}{z_{\alpha}}_{\text {. }}$

Notice that

$$
T C E_{Z}\left(z_{\alpha}\right)=z_{\alpha}+\mathbb{E}\left(Z-z_{\alpha} \mid Z>z_{\alpha}\right),
$$

where $(Z-t \mid Z>t)$ is known as the residual lifetime in reliability and the excess loss (liability) in finance (Shaked and Shanthikumar, 1994).

The $T C E_{Z}(z)$ function is increasing in $z>0$ or equivalently, $T C E_{Z}(z)$ is decreasing in $\alpha \in(0,1)$, since

$$
\frac{d}{d z}(z-\mathbb{E}(Z-z \mid Z>z)) \geq 0
$$

Journal home page: www.jafristat.net 
B.O. Osu, S.A. Ihedioha and E. Ekuma-Okereke, Afrika Statistika, Vol. 8, 2013, pages 545-559.

The price of portfolio selection under tail conditional expectation with consumption cost and

transaction cost.

However in some dynamical settings, it is possible to transform a TCE limit into an equivalent VaR limit, and conversely (Cuocu, 2008).

Yiu (2004) has successfully controlled risky investment by imposing $V a R$ as a dynamic constraint with a model that applies to the $V a R$ constraint over time and emphasized the repeated re-calculations of the $V a R$ like in practice. He expressed the belief that other risk measures imposed in the same way will achieve similar results. We close that gap here by experimenting with the TCE constraint and extending the utility maximization to cover consumption and terminal wealth.

Akume (2009) studied the dynamic portfolio and consumption choice of a trader subject to a risk limit specified in terms of TCE. The tail conditional expectation is calculated for short intervals of time and imposed as risk constraint dynamically (Akume, 2010).

On the other hand, Davis and Norman (1990) had studied an optimal consumption and investment decisions for an investor who has available a bank account paying a fixed rate of interest and a stock whose price is a log-normal diffusion. They showed that the optimal buying and selling policies are the local times of the two-dimensional process of bank and stock holdings at the boundaries of a wedge-shaped region determined by solving a nonlinear free boundary problem. Osu (2011), obtain the optimal price of an institution's assetsliabilities under the TCE with no transaction cost. This paper extends Osu (2011) by the application of TCE to obtain the price of portfolio selection with consumption cost and transaction cost. Furthermore, we give a two boundary equations in the asset region and the liability region. In the no-transaction region, asset region and liability region, two horizondependent boundaries are characterized to obtain a partial differential equation with close form solution.

The rest of this paper is structured as follows: In section 2, we model the financial market and describe the portfolio dynamics. Section 3, derives the Value-at-Risk $(V a R)$ and tail conditional expectation $(T C E)$ constraints. While section 4 makes precise the portfolio selection problem to be solved.

\section{Formulation of the Problem}

We assume the institution operates on a market of one riskless bank with constant interest rate $r$ and $n$ different stock. The evolution of stock prices is described by an $n$ dimensional Wiener process $W(t)$ on the filtered probability space $\left(\Omega, \mathcal{F},\left(\mathcal{F}_{t}\right), \mathbb{P}\right)$ with $\left(\mathcal{F}_{t}\right)=\sigma\{W(s) ; 0<s<t\}:$

$d B(t)=r B(t) d t$

$d S(t)=\mu_{i} S(t)+\sigma_{i} S(t) d W(t), i=1, \ldots, n$.

Here $\sigma_{t}=\left(\sigma_{i, j}(t)\right)_{i<j<n}$ is an $m \times m$ positive definite matrix representing the covariance structure, $\sigma^{\prime} \sigma$. Where $\sigma^{\prime}$ is the transpose of $\sigma$. The institution has initially $x_{0}$ Naira invested in the bank and $\left(x_{1}, \ldots, x_{n}\right)$ Naira invested in stock $1, \ldots, n$. It can control its portfolio composition by buying and selling arbitrarily large or small amounts of stock from its bank account at any time.

Journal home page: www.jafristat.net 
B.O. Osu, S.A. Ihedioha and E. Ekuma-Okereke, Afrika Statistika, Vol. 8, 2013, pages 545-559. The price of portfolio selection under tail conditional expectation with consumption cost and transaction cost.

The institutions portfolio selection strategy $\theta$ is described by the control processes $C(t)$ and $L(t)$, where $C(t)$ (the institution net cash flow at time $t$ ) and $L(t)$ (the market value of the institution's liabilities at time $t)$ are $f$-adapted vector processes.

The dynamics of the control system (Osu and Ihedioha, 2011) is governed by the differential equations

$$
d B(t)=r B(t) d t-(1+\alpha) d C(t)(1+\lambda) d L(t)
$$

and,

$$
d S(t)=S(t)\left[\left(\mu+\frac{\sigma^{2}}{2}\right) d t+\sigma d W(t)\right]+d C(t)-d L(t),
$$

with boundary conditions $B(t)=B_{t}, B(0)=B_{0}$, and $S(t)=S_{t}, S(0)=S_{0}$.

Defined a wealth process $h(t)$ as a sum additive random and multiplicative terms thus:

$$
h(t)=\left\{\begin{array}{l}
(1-\lambda) S(t)+B(t), \text { with probability } q \\
(1-\lambda) S(t) B(t), \text { with probability } 1-q
\end{array}\right.
$$

where $\lambda$ is a stochastic positive factor with probability distribution $\pi(\lambda)$, such that with probability $q$ the integral form of (1) and (2) combined is

$$
h(t)=h(0)+\int_{0}^{t}\left[r B+(1-\lambda)\left(\mu+\frac{\sigma^{2}}{2}\right)\right] d s+(1-\lambda) \sigma \int_{0}^{t} d W(s)-(1+\alpha) C(t) .
$$

The processes, $C(t), L(t)$ and hence $h(t)$ are right continuous with left limit at each $t \geq 0$. For each available strategy $(C, L)$, we can associate a feasible set of controls of the long term performance functional

$$
\mathfrak{F}_{z}(C, L)=\lim _{t \rightarrow \infty} \frac{1}{t} \mathbb{E}_{x}[\ln (h(t))]
$$

with $z=(C, L), z \in \mathbb{R}_{+}^{2}$.

The objective is to optimize the long-run rate of growth

$$
V(x, y)=\sup _{(C, L) \in \mathcal{B}} \mathfrak{F}_{z}(C, L) .
$$

$\mathcal{B}$ is a class of pair $(x, y) \in z$, where $x$ and $y$ are the initial endowment of the riskless and risky asset respectively.

Let $(C, L)$ be any feasible policy. These set of controls can be approximated by a sequence of continuous processes $\left(C_{n}, L_{n}\right)$, such that for $h_{n}$ the net wealth corresponding to them, we have;

$$
\lim _{t \rightarrow \infty} \inf \frac{1}{t}[\ln h(t)] \leq \lim _{n} \lim _{t \rightarrow \infty} \inf \frac{1}{t}\left[\ln h_{n}(t)\right] .
$$

Thus, we can softly assume $\left(C_{n}, L_{n}\right)$ such that for the wealth corresponding to them, we have $\left(C_{0}=L_{0}=0\right)$. 
B.O. Osu, S.A. Ihedioha and E. Ekuma-Okereke, Afrika Statistika, Vol. 8, 2013, pages 545-559. The price of portfolio selection under tail conditional expectation with consumption cost and transaction cost.

Applying Ito's formula, the relationship between $\ln h(t)$ and the processes $B(t)$ and $S(t)$ is (Rodriguez-Pedraza, 2005);

$$
\begin{gathered}
\ln h(t)=\int_{0}^{t} \frac{1}{h(s)}\left[r B(s)+(1-\lambda)\left(\mu+\frac{\sigma^{2}}{2}\right) S(s)\right] d s \\
+(1-\lambda) \sigma \int_{0}^{t} \frac{S(s)}{h(s)} d W(s)-(\lambda-\alpha) \int_{0}^{t} \frac{d C(s)}{h(s)}
\end{gathered}
$$

when $\lambda \rightarrow 0$ and $\alpha \rightarrow 0$, obtain the case of no consumption and transaction costs as in Osu (2011).

Let $\lambda=\alpha$, Equation (5) becomes;

$$
\ln h(t)=\int_{0}^{t} \frac{1}{h(s)}\left[r B(s)+(1-\lambda)\left(\mu+\frac{\sigma^{2}}{2}\right) S(s)\right] d s+(1-\lambda) \sigma \int_{0}^{t} S(s) d W(s),
$$

or

$$
h(t)=h_{0} \exp \int_{0}^{t}\left[r B(s)+(1-\lambda)\left(\mu+\frac{\sigma^{2}}{2}\right) S(s)\right] d s+(1-\lambda) \sigma \int_{0}^{t} S(s) d W(s),
$$

where $h_{0}>0$ denotes the initial value of the portfolio.

This implies,

$$
h(t+\tau)=\exp \left\{\int_{t}^{t+\tau}\left[r B(s)+(1-\lambda)\left(\mu+\frac{\sigma^{2}}{2}\right) S(s)\right] d s+(1-\lambda) \sigma \int_{t}^{t+\tau} S(s) d W(s)\right\} .
$$

For a given $\tau>0, h>0$, and $S \in \mathbb{R}^{n}$, we have

$$
h_{t+\tau}=h(t) \exp \left(\left(r B(s)+(1-\lambda)\left[\mu+\frac{\sigma^{2}}{2}\right] S(s)\right) \tau+(1-\lambda) \sigma S(w(t+\tau)-w(t))\right) .
$$

Now for a given probability level $\lambda \in(0,1)$ and a given horizon $\tau>0$, the $V a R$ at time $t$ of a portfolio $s \in S$, denoted by $\operatorname{VaR}_{t}^{\lambda, s}$ is given by:

$$
V a R_{t}^{\lambda, s}=\inf \left\{L \geq 0, \mathbb{P}\left(h_{t}^{s}-h_{t+\tau}\left(h_{t}^{s}, S_{t}\right)\right) \geq L \mid f_{t}<\lambda\right\}=\left(Q_{t}^{\lambda, s}\right)^{-},
$$

where

$$
\left(Q_{t}^{\lambda, s}\right)^{-}=\sup \left\{L \in \mathbb{R}^{n}:\left\{\mathbb{P}\left(h_{t}^{s}-h_{t+\tau}\left(h_{t}^{s}, S_{t}\right)\right) \geq L \mid f_{t}<\lambda\right\}\right\}
$$

is the quantile of the projected asset gain over the interval $(t, t+\tau)$ and $z^{-}=\max [0,-z]$. In other words, $\operatorname{VaR}_{t}^{\lambda, S}$ is the liability over the next period of length $\tau$ which would be exceeded only with a (small) conditional probability $\lambda$ if the current price $S_{t}$ was kept unchanged.

The fact that $V a R_{t}^{\lambda, S}$ is computed under the assumption that the current portfolio is kept unchanged reflects the actual practice and the fact that the financial institutions monitoring 
B.O. Osu, S.A. Ihedioha and E. Ekuma-Okereke, Afrika Statistika, Vol. 8, 2013, pages 545-559. The price of portfolio selection under tail conditional expectation with consumption cost and transaction cost.

their traders do not typically know the trades' future portfolio choices over $V a R$ horizon. The measure of $V a R$ in (6) only requires the knowledge of the current portfolio value, the current asset value and the conditional distribution of asset returns.

The $T C E$ of a price $s \in S$ is defined by;

$$
T C E_{t}^{\lambda, s}=\left(\frac{\mathbb{E}\left[h_{t}^{s}-h_{t+\tau}\left(h_{t}^{s}, S_{t}\right)\right] h_{t}^{s}-h_{t+\tau}\left(h_{t}^{s}, S_{t}\right) \geq-Q_{t}^{\lambda, s}}{\lambda} \mid f_{t}\right)^{+},
$$

where $z^{+}=\max \{0, z\}$.

\section{Value-at-Risk and tail conditional expectation constraints}

3.1. Value-at-Risk and tail conditional expectation constraints when, $\lambda=\alpha$

Lemma 1. Let $N(x)$ and $N^{-1}(x)$ denote the normal distribution and inverse normal distribution functions with

$$
\epsilon=(1-\lambda)\left[\mu+\frac{\sigma^{2}}{2}\right]
$$

and

$$
\phi=(1-\lambda)
$$

Then,

$$
V a R_{t}^{\lambda, s}=h_{t}^{s}\left[1-\exp (r B(s)+\epsilon S(s)) \tau+\phi N^{-1}(\lambda) S_{t} \sigma \sqrt{\tau}\right]^{+}
$$

and

$$
T C E_{t}^{\lambda, s}=h_{t}^{s}\left[1-\exp \frac{(r B(s)+\epsilon S(s)) \tau+N N^{-1}(\lambda)+\sigma \sqrt{\tau} S_{t}}{\lambda}\right]^{+} .
$$

Proof. We have

$$
\begin{aligned}
& \mathbb{P}\left(h_{t+\tau}\left(h_{t}, S_{t}-h_{t}\right) \leq \mid f_{t},\right) \\
= & \mathbb{P}\left(\exp \left(r B(s)+\epsilon S(s)+\phi S(w(t+\tau)-w(t)) \leq 1+\frac{L}{h_{t}} \mid f_{t},\right)\right) \\
= & \left.\mathbb{P}\left(\sigma S(w(t+\tau)-w(t)) \leq \frac{\ln \left(1+\frac{L}{h_{t}}\right)^{+}-(r B(s)+\epsilon S(s)) \tau}{\phi}\right) f_{t},\right) \\
= & N\left(\frac{\ln \left(1+\frac{L}{h_{t}}\right)^{+}-(r B(s)+\epsilon S(s)) \tau}{\phi}\right) .
\end{aligned}
$$

The last equation is due to the fact that the random variable $\phi \sigma S(w(t+\tau)+w(t))$ is conditionally normally distributed.

Thus

$$
\left.\mathbb{P}\left(h_{t+\tau}\left(h_{t}\right), S_{t}-h_{t}\right) \leq \mid f_{t}\right) \leq \lambda
$$


B.O. Osu, S.A. Ihedioha and E. Ekuma-Okereke, Afrika Statistika, Vol. 8, 2013, pages 545-559. The price of portfolio selection under tail conditional expectation with consumption cost and transaction cost.

$$
\begin{gathered}
\Leftrightarrow N\left(\frac{\ln \left(1+\frac{L}{h_{t}}\right)^{+}-(r B(s)+\epsilon S(s)) \tau}{\phi}\right) \\
\Rightarrow L \leq h(t)\left[\exp \left\{(r B(s)+\epsilon S(s)) \tau+\phi N^{-1}(\lambda) S_{t} \sigma \sqrt{\tau}\right\}-1\right]^{+},
\end{gathered}
$$

which implies;

$$
Q_{t}^{\lambda, s}=h_{t}^{s}\left\{\exp \left[(r B(s)+\epsilon S(s)) \tau+\phi N^{-1}(\lambda) S_{t} \sigma \sqrt{\tau}\right\}-1\right]^{+} .
$$

Therefore,

$$
V a R_{t}^{\lambda, s}=h_{t}^{s}\left\{1-\exp (r B(s)+\epsilon S(s)) \tau+\phi N^{-1}(\lambda) S_{t} \sigma \sqrt{\tau}\right\}^{+}
$$

Also,

$$
\begin{aligned}
& \mathbb{E}\left\{\left(h_{t}^{s}-h_{t+\tau}\left(h_{t}^{s}, S_{t}\right)\right)\left[h_{t}^{s}-h_{t+\tau}\left(h_{t}^{s}, S_{t}\right)\right]-Q_{t}^{\lambda, s}(t) \mid f_{t}\right\} \\
= & h_{t}^{s} \mathbb{E}\left\{-\exp \left(\left(r B(t)+\epsilon S_{t}\right) \tau+\phi \sigma S_{t}(w(t+\tau)-w(t)) \frac{\phi \sigma S_{t}(w(t+\tau)-w(t))}{\sigma \sqrt{\tau} S_{t}} \leq \phi N^{-1}(\lambda) \mid f_{t}\right)\right\} \\
= & h_{t}^{s}\left\{\lambda-\exp \left(\left(r B(t)+\epsilon S_{t}\right) \tau\right) \int_{-\infty}^{\phi N^{-1}(\lambda)} \frac{1}{2 \pi} \exp \left(-\frac{\left(x-\sigma \sqrt{\tau S_{t}}\right)^{2}}{2}\right) d x\right\} \\
= & h_{t}^{s}\left\{\lambda-\exp \left(\left(r B(t)+\epsilon S_{t}\right) \tau\right) N\left(\phi N^{-1}(\lambda)-\sigma \sqrt{\tau S_{t}}\right)\right\} .
\end{aligned}
$$

Particulary,

$$
0 \leq V a R_{t}^{\lambda, s} \leq T C E_{t}^{\lambda, s} \leq h_{t}^{s} \text { and } V a R_{t}^{\lambda, 0} \leq T C E_{t}^{\lambda, 0}=0 .
$$

We seek the asset and liability allocation that maximizes (over admissible $\left(C_{t}, L_{t}\right)$ ) the expected utility of terminal wealth at time $T$ and liability over the entire horizon $[0, T]$, for a risk averse institution that limits its risk by imposing an upper bound on the TCE.

In mathematical terms the stochastic asset-liability control problem with transaction under a TCE constraint is:

$$
\max _{(C, L) \in \mathcal{B}} \mathbb{E}\left(U\left(h_{t}^{s}\right)\right)
$$

subject to the wealth process

$$
\begin{gathered}
h_{t}^{s}=h_{0} \exp \int_{0}^{t}[r B(s)+\epsilon S(s)] d s+\phi \int_{0}^{t} S(s) d W(s), \\
\ln \left(\frac{1-\overline{T C E}\left(h_{t}^{s}, t\right)}{h_{t}^{s}}\right)-\left(r B(t)+\epsilon S_{t}\right) \tau+N\left(\phi N^{-1}(\lambda) S_{t} \sigma \sqrt{\tau}\right) \leq 0
\end{gathered}
$$

and the $T C E$ constraint for fixed $\Delta t>0$ given by

$$
T C E_{t}^{\lambda, s} \leq \rho(h, t), \forall t \in[0, \tau] .
$$

Where

$$
\rho\left(S_{t}, t\right)=1-\exp \left(r B(t)+\epsilon S_{t}\right) \tau+N\left(\phi N^{-1}(\lambda) S_{t} \sigma \sqrt{\tau}\right)
$$


B.O. Osu, S.A. Ihedioha and E. Ekuma-Okereke, Afrika Statistika, Vol. 8, 2013, pages 545-559. The price of portfolio selection under tail conditional expectation with consumption cost and transaction cost.

and

$$
\hat{\rho}\left(S_{t}, t\right)=1-\exp \left(r B(t)+\phi S_{t}\right) \tau \frac{N\left(\phi N^{-1}(\lambda)-\sigma \sqrt{\tau S_{t}}\right)}{\hat{\lambda}} .
$$

With probability $1-q$, we have

$$
h(t)=(1-\lambda) S(t) B(t)
$$

or

$$
S(t)=\frac{h(t)}{(1-\lambda) B(t)}
$$

This is based on the classical function which implies that the price $S(t)$ of the risky asset equals the ratio of multiple of the wealth process $h(t)$ to the price of bond $B(t)$.

Applying the TCE constraint while maximizing the institution's logarithmic utility over asset-liabilities throughout the investment horizon and over the terminal wealth, we have:

$$
\max _{(C, L) \in \mathcal{B}} \mathbb{E}\left(U\left(h_{t}^{s}\right)\right)
$$

subject to the wealth process;

$$
\begin{gathered}
h_{t}^{s}=h_{0} \exp \int_{0}^{t}\left[r B(s)+\beta \frac{h(s)}{B(s)}\right] d s+\sigma \int_{0}^{t} \frac{h(s)}{B(s)} d W(s), \\
\ln \left((1-\lambda)-\frac{\overline{T C E}\left(h_{t}^{s}, t\right)}{(1-\lambda) S(t) B(t)}\right)-\left(r B(t)+\beta \frac{h(t)}{B(t)}\right) \tau \\
+\ln N \phi N^{-1}(\lambda) \frac{h(t)}{B(t)} \sigma \sqrt{\tau} \leq 0,
\end{gathered}
$$

with $\beta=\left[\mu+\frac{\sigma^{2}}{2}\right]$.

\subsection{Value-at-Risk and tail conditional expectation constraints when, $\lambda \neq \alpha$}

Using (5) with $\epsilon=(1-\lambda)\left[\mu+\frac{\sigma^{2}}{2}\right], \phi=(1-\lambda), \beta=\left[\mu+\frac{\sigma^{2}}{2}\right]$ and $\eta=(\lambda+\alpha)$, we obtain:

$$
h(t)=h_{0} \exp \int_{0}^{t}[r B(s)+\epsilon S(s)] d s+\phi \int_{0}^{t} S(s) d W(s)+\eta C(t)
$$

and

$$
h(t+\tau)=\exp \left\{\int_{0}^{t+\tau}[r B(s)+\epsilon S(s)] d s+\phi \int_{0}^{t+\tau} S(s) d W(s)\right\}+\eta C(t)
$$

for any $\tau>0$.

So that

$$
h_{t+\tau}=h(t) \exp ((r B(s)+\epsilon S(s)) \tau+\phi \sigma S(w(t+\tau)-w(t)))+\eta C(t)
$$


B.O. Osu, S.A. Ihedioha and E. Ekuma-Okereke, Afrika Statistika, Vol. 8, 2013, pages 545-559.

The price of portfolio selection under tail conditional expectation with consumption cost and transaction cost.

Lemma 2. The value-at-risk $V a R$ and Tail conditional expectation are given as follows:

$$
V a R_{t}^{\alpha, s}=h_{t}^{s}\left[1-\exp (r B(s)+\epsilon S(s)) \tau+\eta C(t)+\phi N^{-1}(\alpha)\right]^{+}
$$

and

$$
T C E_{t}^{\alpha, s}=h_{t}^{s}\left[1-\exp \frac{(r B(s)+\epsilon S(s)) \tau+\eta C(t)+N\left(\phi N^{-1}(\alpha)\right)}{\alpha}\right]^{+},
$$

where $N(x)$ and $N^{-1}(x)$ denote the normal distribution and inverse normal distribution functions.

Proof. Following similar method of proof as in lemma 1, we have

$$
\begin{aligned}
& \mathbb{P}\left(h_{t+\tau}\left(h_{t}, S_{t}-h_{t}\right) \leq \mid f_{t},\right) \\
= & \mathbb{P}\left(\exp (r B(s)+\epsilon S(s)) \tau+\eta C(t)+\phi \sigma S(w(t+\tau)-w(t)) \leq 1+\frac{L}{h_{t}} \mid f_{t}\right) \\
= & \mathbb{P}\left(\sigma S(w(t+\tau)-w(t)) \leq \frac{\ln \left(1+\frac{L}{h_{t}}\right)^{+}-\exp (r B(s)+\epsilon S(s)) \tau-\eta C(t)}{\phi} \mid f_{t},\right) \\
= & N\left\{\frac{\ln \left(1+\frac{L}{h_{t}}\right)^{+}-(r B(s)+\epsilon S(s)) \tau-\eta C(t)}{\phi}\right\} .
\end{aligned}
$$

The last equation is due to the fact that the random variable $\phi(w(t+\tau)-w(t))$ is conditionally normally distributed with mean 0 and variance $S\left(t, \phi^{2}\right) \tau$.

Thus

$$
\begin{gathered}
\mathbb{P}\left(h_{t+\tau}\left(h_{t}\right), S_{t}-h_{t} \leq \mid f_{t}\right) \leq \alpha \\
\Leftrightarrow N\left\{\frac{\ln \left(1+\frac{L}{h_{t}}\right)^{+}-(r B(s)+\epsilon S(s)) \tau-\eta C(t)}{\phi}\right\} \\
\Rightarrow L \leq h(t)\left[\exp \left\{(r B(s)+\epsilon S(s)) \tau+\eta C(t)+\phi N^{-1}(\alpha) \sigma S_{t} \sigma \sqrt{\tau}\right\}-1\right]^{+}
\end{gathered}
$$

which implies:

$$
Q_{t}^{\lambda, s}=h_{t}^{s}\left\{\exp \left[(r B(s)+\epsilon S(s)) \tau+\eta C(t)+\phi N^{-1}(\alpha) S_{t} \sigma \sqrt{\tau}\right]-1\right\}^{+} .
$$

Therefore,

$$
V a R_{t}^{\lambda, s}=h_{t}^{s}\left\{1-\exp (r B(s)+\epsilon S(s)+\eta C(s)) \tau+N^{-1}(\alpha) \sigma \sqrt{\tau S_{t}}\right\}^{+} .
$$

Similarly

$$
\mathbb{E}\left\{\left(h_{t}^{s}-h_{t+\tau}\left(h_{t}^{s}, S_{t}\right)\right)\left[\left(h_{t}^{s}-h_{t+\tau}\left(h_{t}^{s}, S_{t}\right)\right)\right] z-Q_{t}^{\lambda, s}(t) \mid f_{t}\right\}
$$


B.O. Osu, S.A. Ihedioha and E. Ekuma-Okereke, Afrika Statistika, Vol. 8, 2013, pages 545-559. The price of portfolio selection under tail conditional expectation with consumption cost and transaction cost.

$$
\begin{gathered}
=h_{t}^{s} \mathbb{E}(-\exp ((r B(s)+\epsilon S(s)) \tau+\eta C(t) \\
\left.\left.+\phi S_{t}(w(t+\tau)-w(t)) \frac{\phi S_{t}(w(t+\tau)-w(t))}{\sigma \sqrt{\tau S_{t}}} \leq N^{-1}(\alpha) \mid f_{t}\right)\right) \\
=h_{t}^{s}\left\{\alpha-\exp ((r B(s)+\epsilon S(s)) \tau+\eta C(t)) \int_{-\infty}^{N^{-1}(\lambda)} \frac{1}{2 \pi} \exp \left(-\frac{\left(x-\sqrt{\tau S_{t}}\right)^{2}}{2}\right) d x\right\} \\
=h_{t}^{s}\left\{\alpha-\exp ((r B(s)+\epsilon S(s)) \tau+\eta C(t)) N\left(\phi N^{-1}(\alpha)-\phi \sigma \sqrt{\tau S_{t}}\right)\right\} .
\end{gathered}
$$

The stochastic asset-liability control problem with transaction under a TCE constraint is now:

$$
\max _{(C, L) \in \mathcal{B}} \mathbb{E}\left(U\left(h_{t}^{s}\right)\right)
$$

subject to the wealth process

$$
\begin{gathered}
\exp \int_{0}^{t}[r B(s)+\epsilon S(s)] d s+\eta C(t)+\phi \int_{0}^{t} S(s) d W(s), \\
\ln \left(\frac{1-\overline{T C E}\left(h_{t}^{s}, t\right)}{h_{t}^{s}}\right)-r B(s)+\epsilon S(s)+\eta C(t)+N\left(\phi N^{-1}(\alpha) \sigma \sqrt{\tau S(t)}\right) \leq 0 .
\end{gathered}
$$

For fixed $\Delta t>0$, the TCE constraint is given by,

$$
T C E^{\alpha, s} \leq \rho(h, t), \forall t \in[0, \tau],
$$

where

$$
\rho\left(S_{t}, t\right)=1-\exp \left(r B(t)+\epsilon S_{t}+\eta C(s) \tau+N\left(\phi N^{-1}(\alpha) \sigma \sqrt{\tau S(t)}\right)\right.
$$

and

$$
\hat{\rho}\left(S_{t}, t\right)=1-\exp \left(r B(t)+\epsilon S_{t}+\eta C(s) \tau+\frac{N\left(\phi N^{-1}(\alpha) \sigma \sqrt{\tau S(t)}\right.}{\hat{\alpha}}\right)
$$

The application of the TCE constraint now gives:

$$
\max _{(C, L) \in \mathcal{B}} \mathbb{E}\left(U\left(h_{t}^{s}\right)\right)
$$

subject to the wealth process;

$$
\begin{gathered}
h_{t}^{s}=h_{0} \exp \int_{0}^{t}\left[r B(s) \beta \frac{h(s)}{B(s)}+\eta(s)\right] d s+\sigma \int_{0}^{t} \frac{h(s)}{B(s)} d W(s), \\
\ln \left((1-\lambda)-\frac{\overline{T C E}\left(h_{t}^{s}, t\right)}{(1-\lambda) S(t) B(t)}\right)-\left(r B(t)+\rho \frac{h(t)}{B(t)}\right) \tau+N\left(\phi N^{-1}(\alpha) \sigma \sqrt{\tau}\right) \frac{h(s)}{B(s)} \leq 0 .
\end{gathered}
$$


B.O. Osu, S.A. Ihedioha and E. Ekuma-Okereke, Afrika Statistika, Vol. 8, 2013, pages 545-559. The price of portfolio selection under tail conditional expectation with consumption cost and transaction cost.

\section{The Price of Portfolio Selection under TCE with Consumption cost and no Transaction Cost}

The asset-liability problem is:

$$
J(C, B, S, t ; T)=\max _{C_{t}, B_{t}, S_{t}>0} \mathbb{E}\left[\int_{0}^{T} e^{-\rho \tau \frac{C_{t} 1-\gamma}{1-\gamma}} d \tau+e^{-\rho T \frac{\left(B_{T}+S_{T}\right)^{1-\gamma}}{1-\gamma}}\right] .
$$

Subject to:

$$
\begin{aligned}
& d B(t)=r B(t)-d C(t)+(1-\lambda) d L(t) \\
& d S(t)=S(t)\left[\left(\mu+\frac{\sigma^{2}}{2}\right) d t+\sigma d W(t)\right]+d C(t)-d L(t) .
\end{aligned}
$$

The value function should also satisfy the terminal condition, that all the stock holding must be transformed to cash at time $T$ :

$$
J(C, B, S, t ; T)=\frac{\left(B_{T}+S_{T}\right)^{1-\gamma}}{1-\gamma} .
$$

Assumption 1. The value function $J(C, B, S, t ; T)$ is once continuously differentiable in $B$ and twice continuously differentiable in $S$. The two boundary equations in the asset region and the liability region are as given below. In the no-transaction region, to obtain the HJB, apply Ito's lemma:

$$
d J=\left(\frac{d J}{d t}+(r B-C) \frac{d J}{d B}+\mu S \frac{d J}{d S}+\frac{1}{2} S^{2} \frac{d^{2} J}{d S^{2}}\right) d t+\sigma S \frac{d J}{d s} d Z,
$$

to the Bellman equation (7). We have:

$$
\begin{gathered}
\frac{C^{1-\gamma}}{1-\gamma} J_{t}+J_{B}(r B-C)+J_{S} \mu S+\frac{1}{2} J_{S S} \sigma^{2} S^{2}-\rho J=0,: 0 \leq \frac{B}{S} \leq P, \\
J_{B}=(1-q) J_{S},: \frac{B}{S}>P, \\
(1-q) J_{B}=J_{S},: \frac{B}{S}<0 .
\end{gathered}
$$

Substituting optimal consumption into HJB equation $C^{*}=\left(J_{B}\right)^{-1 / \gamma}$ yields:

$$
\frac{\left(J_{B}^{\frac{-1}{\gamma}}\right)^{1-\gamma}}{1-\gamma}+J_{t}+\left(r B-J_{B}^{\frac{-1}{\gamma}}\right) J_{B}+\mu S J_{S}+\frac{1}{2} \sigma^{2} S^{2} J_{S}-\rho J=0 ;: 0 \leq \frac{B}{S} \leq P
$$

The value function $J(C, B, S, t ; T)$ is homogeneous of degree $1-\gamma$ for all positive numbers in $(B, S)$, as shown in Fleming and Soner (1993)xxx. Define $h=\frac{B}{S}$, for a new value function

$$
f:(-\infty,+\infty) \times[0, T] \rightarrow \mathbb{R} .
$$

Homogeneity gives:

$$
J(C, B, S, t ; T)=S^{1-\gamma} f(C, h, t ; T) .
$$


B.O. Osu, S.A. Ihedioha and E. Ekuma-Okereke, Afrika Statistika, Vol. 8, 2013, pages 545-559. The price of portfolio selection under tail conditional expectation with consumption cost and transaction cost.

The no-transaction region, asset region and liability region thus can be characterized by two horizon-dependent boundaries $O(t ; T)$ and $P(t ; T)$. We derive the new value function and its derivatives with respect to $h$ and $t$ by applying the chain rule, as follows:

$$
\begin{gathered}
J=S^{1-\gamma} f, J_{t}=S^{1-\gamma} f_{t}, J_{B}=S^{-\gamma} f_{h}, J_{S}=(1-\gamma) S^{-\gamma} f-B S^{-\gamma-1} f_{h}, \\
J_{S S}=\gamma(1-\gamma) S^{-\gamma-1} f+2 \gamma B S^{-\gamma-2} f_{h}+B^{2} S^{-\gamma-3} f_{h h} .
\end{gathered}
$$

Substituting the new value function and its derivatives with respect to $\mathrm{h}$ into the modified HJB equation and the two boundary equations on the asset region and the liability region, one obtains a system of partial differential equations (PDEs).

On the no-transaction region:

$$
\begin{gathered}
\frac{\gamma}{1-\gamma} f_{h}^{1-\frac{1}{\gamma}}+\frac{1}{2} f_{h h} \sigma^{2} h^{2}+f_{h}\left(\gamma \sigma^{2}-(\mu-r]\right) h+f\left((1-\gamma)\left(\mu-\frac{\gamma \sigma^{2}}{2}\right)-\rho\right)+f_{t}=0 \\
O(t ; T) \leq h \leq P(t ; T) .
\end{gathered}
$$

For

$$
\frac{\gamma}{1-\gamma} \gamma f_{h}^{\frac{-1}{\gamma}}=(\gamma-1) \iota h
$$

(9) becomes:

$$
\begin{gathered}
\frac{1}{2} f_{h h} \sigma^{2} h^{2}+f_{h}\left(\gamma \sigma^{2}-(\mu-r)+(\gamma-1) \mu\right) h+f\left((1-\gamma)\left(\mu-\frac{\gamma \sigma^{2}}{2}\right)-\rho\right)+f_{t}=0 \\
O(t ; T) \leq h \leq P(t ; T) .
\end{gathered}
$$

On the asset region:

$$
\left(\frac{1}{1-q}+h\right) f_{h}(h, t ; T)=(1-\gamma) f(h, t ; T) ;: h>P(t, T) .
$$

On the liability region:

$$
(1-q+h) f-h(h, t ; T)=(1-\gamma) f(h, t ; T) ;: h<O(t, T) .
$$

In addition, the following terminal condition must be satisfied

$$
f(h, t ; T)=\frac{\left(h_{T}+1\right)^{1-\gamma}}{1-\gamma} .
$$

Theorem 1. Let $f(h)$ be the price of the asset-liability with $h$ as the prevailing money market account-stock ratio. Let $f(h)$ be twice continuously differentiable, the solution of the time-homogeneous value function equation (11) with:

$$
f(0)=0, \text { and } f^{\prime}(h)=0
$$

is given by

$$
f(h)=c(\sigma h)^{\lambda_{1}}+\frac{h}{k-\frac{\xi(t)}{\sigma}},
$$


B.O. Osu, S.A. Ihedioha and E. Ekuma-Okereke, Afrika Statistika, Vol. 8, 2013, pages 545-559. The price of portfolio selection under tail conditional expectation with consumption cost and transaction cost.

with

$$
\frac{\hat{h}}{k-\frac{\xi(t)}{\sigma}}+c \lambda_{1}(\sigma \hat{h})^{\lambda_{1}}=0
$$

and

$$
f(h)=\frac{1}{\xi(t)-\sigma k}\left\{\frac{\sigma^{1+\lambda_{1}} h^{1-\lambda_{1}}}{\lambda_{1}}+\sigma h\right\},
$$

where $\hat{h}$ is the expected optimal money market account-stock ratio for a period $t, c$ is a constant and;

$$
\begin{aligned}
& \lambda_{1}=-\left[\frac{\xi}{\sigma}-\frac{1}{2}\right]+\left\{\left[\frac{\xi}{\sigma}-\frac{1}{2}\right]^{2}+2 k\right\}^{\frac{1}{2}} \\
& \lambda_{2}=-\left[\frac{\xi}{\sigma}-\frac{1}{2}\right]-\left\{\left[\frac{\xi}{\sigma}-\frac{1}{2}\right]^{2}+2 k\right\}^{\frac{1}{2}},
\end{aligned}
$$

are the positive and negative characteristic roots of (8) respectively.

Proof. Let $\xi=\left(\gamma \sigma^{2}-(\mu-r)+(\gamma-1) \mu\right)$ and $k=\left\{(\gamma-1)\left(\mu-\frac{\gamma \sigma^{2}}{2}\right)+\rho\right\}$, then (10) reduces to the ode (with the conditions in Osu and Okoroafor, 2007)

$$
\frac{\sigma^{2} h^{2}}{2} f_{h h}+\xi h f_{h}-k f=-h .
$$

By the method of change of independent variables using Euler's substitution and solving by variation of parameters, the solutions are obtained. An important relationship derived under the optimal condition is that the discount rate is proxy of the systematic volatility factor in the economy. So that the discounted rate gains from a unit investment at $\hat{h}$ equals the optimal unit $\bar{h}$ of ratio of money market account to stock for the expected optimal money market account to stock ratio $\hat{h}$. Therefore, by (14), we have

$$
f(\hat{h})=c(\sigma \hat{h})^{\lambda_{1}}+\frac{\hat{h}}{k-\frac{\xi}{\sigma}}=\bar{h} .
$$

Solving for $\mathrm{C}$ in (16) and (21) and equating the results gives:

$$
\hat{h}=\frac{\lambda_{1}\left[k-\frac{\xi}{\sigma}\right]}{\lambda_{1}-1} .
$$

Note: When the drift parameter $\xi$ is large enough so that $\frac{\xi}{\sigma}>\frac{1}{2}$ then the right hand side of (18) is approximated by first order Taylor's expansion as $\frac{k}{\frac{\xi}{\sigma}-\frac{1}{2}}$, thus, $\frac{\xi}{\sigma}-\frac{1}{2}$ and we obtain $\lambda_{1}=1$ and the optimal money market account-stock ratio of (22) becomes indeterminate. 
B.O. Osu, S.A. Ihedioha and E. Ekuma-Okereke, Afrika Statistika, Vol. 8, 2013, pages 545-559. The price of portfolio selection under tail conditional expectation with consumption cost and transaction cost.

\section{5. conclusions}

We have considered in this paper the Price of Portfolio Selection under Tail Conditional Expectation with Consumption cost and Transaction Cost. The dependence of expected return and $T C E$ on the initial portfolio, in particular when transaction costs are high, is largely removed by the introduction of the $T C E$ constraint. In answer to the second question our analysis shows that both expected return and risk as measured by TCE are brought to some intermediate level when intermediate transactions are made possible subject to a $T C E$ constraint. Without the TCE constraint, intermediate transactions aiming at maximising expected log-returns lead to higher returns and higher risk when initial portfolios are low return. Notice that on the asset and liability regions, $\frac{1}{1-q}+h=1-q+h$, which gives $q=0$ or $q=2$. Equation (11) has the solution, $f(h)=k\left(\frac{1}{1-q}+h\right)^{1-\gamma}$ which becomes $f(h)=k(1+h)^{1-\gamma}$ for $q=0$ and $f(h)=k(h-1)^{1-\gamma}$ for $q=2$. For $k>0, f(h)$ increases as $q \rightarrow 0$ and decreases as $q \rightarrow 2$. Thus if there is no transaction cost, growth rate of the value function is higher than when there is transaction cost. On the other hand, equation (12) has a solution; $f(h)=k(1-q+h)^{1-\gamma}$. Generally, the value of the investment increases (decrease) in the asset region and decreases (increases) in the liability region for some values of $0<q<2$, but are equal when $q=0$ or $q=2$.

\section{References}

Aït-Sahalia, Y., 2001. Variable Selection for Portfolio Choice. Journal of Finance, American Finance Association, 56(4).1297-1351.

Akume, D., Luderer, B. and Wunderlich, R., 2009. Optimal portfolios under dynamic shortfall constraints. Afr. Stat. 4. 156-167.

Akume, D., Luderer, B. and Wunderlich, R., 2010. Dynamic shortfall constraints for optimal portfolios. Surveys in Mathematics and its Applications. 5, pp.135-149.

Artzner, F.D., Eber, J.M. and Heath, D., 1999. "Coherent measures of Risk." Mathematical Finance, 9(3). 203-228.

Cuocu , D., He, H. and Issaenko, S., 2008. Optimal Dynamic Trading Strategies with Risk limits. Operations Research, 56: 356-368. (April 1.2001). http://ssrn.com/abstract $=563901$.

Davis, M.H.A. and Norman, A.R., 1990. Portfolio Selection with transaction costs. Mathematics of operation Research. 15(4), 676-713.

Landsman, Z. and Valdez, E., 2003. Tail conditional expectations for elliptical distributions. American Actuarial J. 7, 55-71.

Lan, H., Nelson, B.L. and Staum, J., 2010. A Confidence Interval Procedure for Expected Shortfall Risk Measurement via Two-Level Simulation. Operations Research. 58(5), 14811490.

Jorion, P., 2001. Value at Risk. McGraw-Hill publishers. New York.

Markowitz, H.M., 1952. Portfolio selection. The Journal of Finance. 7(1), 77-91.

McNeil, A.J., Fleming, W.H. and Soner, H.M., 1993. Controlled Markov Processes and Viscosity Solutions. Springer Verlag, New York.

Frey, R. and Embrechts, P., 2006. Quantitative Risk Management: Concepts Techniques and Tools. Princeton University Press. 0980.91042. 
B.O. Osu, S.A. Ihedioha and E. Ekuma-Okereke, Afrika Statistika, Vol. 8, 2013, pages 545-559. The price of portfolio selection under tail conditional expectation with consumption cost and transaction cost.

Osu, B.O., 2011. The price of Asset-liability Control under Tail Conditional Expectation with No Transaction Costs. British Journal of Mathematics \& Computer Science, 1(3), 129-140.

Osu, B.O. and Ihedioha, S.A., 2011. Use of Stochastic Asset-Liability Model to Find Unique Price of Asset. British Journal of Mathematics \& Computer Science, 1(2), 101-11.

Osu, B.O. and Okoroafor, A.C.,(2007). On the Measurement of Random Behaviour of Price Changes. Journal of Mathematical Sciences, 18(2), 131-141.

Rockafellar, R.T. and Uryasev, S., 2001. Conditional Value-at-risk for general loss distributions. Research report 2001-5 (downloaded: www.ise.ufl.edu/uryasev/cvar2.pdf).

Rodriguez-Pedraza, R.A., 2005. Optimal portfolio allocation for long-term growth of wealth in presence of transaction costs. M.Sc. thesis, Department of Applied Mathematics. Iowa State University.

Shaked, M. and Shanthikumar, J.G., 1994. Stochastic Orders and their Applications. Academic Press. New York.

Yiu, K.F.C. (2004). Optimal portfolios under a value at risk constraint. Journal of Economic Dynamics and Control. 28, 1317-1334. 